\title{
Touching the Invisible: Localizing Ultrasonic Haptic Cues
}

\author{
Dong-Bach Vo ${ }^{\star}$ and Stephen A. Brewster ${ }^{\dagger}$ \\ Glasgow Interactive Systems Group, School of Computing Science, University of Glasgow, UK
}

\begin{abstract}
While mid-air gestures offer new possibilities to interact with or around devices, some situations, such as interacting with applications, playing games or navigating, may require visual attention to be focused on a main task. Ultrasonic haptic feedback can provide 3D spatial haptic cues that do not demand visual attention for these contexts. In this paper, we present an initial study of active exploration of ultrasonic haptic virtual points that investigates the spatial localization with and without the use of the visual modality. Our results show that, when providing haptic feedback giving the location of a widget, users perform $50 \%$ more accurately compared to providing visual feedback alone. When provided with a haptic location of a widget alone, users are more than $30 \%$ more accurate than when given a visual location. When aware of the location of the haptic feedback, active exploration decreased the minimum recommended widget size from $2 \mathrm{~cm}^{2}$ to $1 \mathrm{~cm}^{2}$ when compared to passive exploration from previous studies. Our results will allow designers to create better mid-air interactions using this new form of haptic feedback.
\end{abstract}

\section{INTRODUCTION}

Sophisticated and affordable sensors have lead many to consider touchless gestural interaction in new application contexts such as desktop computers, gaming, interactive tabletops or inside cars $[12,14,17]$. In addition to simple tasks such as pan and zoom or switching programs, gestures above the keyboard allow users to manipulate complex widgets without the burden of using another device, such as a mouse. For example, it is possible to enable a colour picker widget with a key on the keyboard and control the wheel with mid-air gestures [17]. Mid-air gestures also provide rich interaction techniques to execute sophisticated commands and give six degrees of freedom with which to manipulate digital content [12]. However, a key limitation with the research and products in this area is that they provide no haptic feedback; users can gesture but they cannot feel the controls they are interacting with.

Gestures above the keyboard can limit the increasing complexity of keyboards by addressing special functionalities such as media controls keys, numeric keypads, specific or additional keyboard layouts, shortcuts, or even specific input controllers like sliders or dials. For example, introducing layers of configurable virtual input controllers in the space around the keyboard would enable a rich set of complementary controllers for secondary or specific tasks (Figure 1). However, this requires the ability to locate such controllers

*dong-bach.vo@glasgow.ac.uk

† stephen.brewster@glasgow.ac.uk easily and without decreasing user performance by cluttering the display with visual information about where they are.

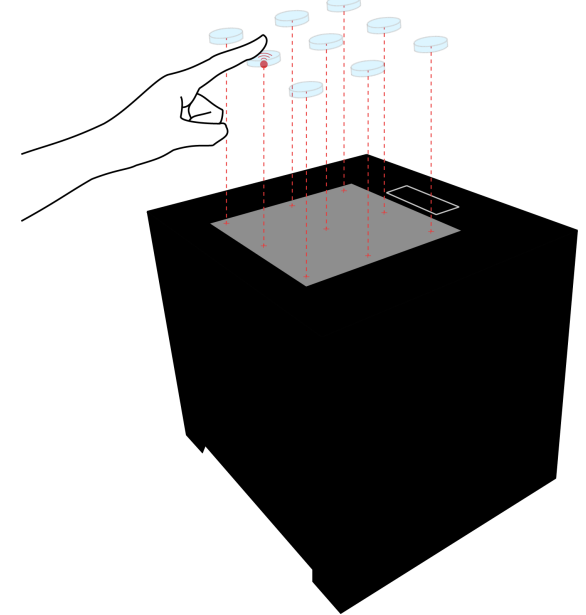

Figure 1. Using ultrasonic haptic feedback to improve performance when interacting with virtual widgets. Widgets are located on the same plane.

In addition of compromising the performance of interacting with computers in an office environment, disengaging visual attention from main tasks can also potentially compromise safety in some contexts. For instance, free-hand pointing has been investigated as a means to select objects of interest outside and on the dashboard of a car for planning a route and storing points of interest in a navigation system while driving [14]. The authors report that the drivers compensated for the decreased of level of visual attention dedicated to the driving task by decreasing their driving speed. In addition, their findings also revealed that locating objects and controlling selections required participants to frequently glance away from traffic, which may have dangerous consequences. Therefore, it is necessary to find a better means for users to use these secondary controls to avoid compromising safety.

Ultrasonic haptic feedback can provide localized continuous haptic cues in $3 \mathrm{D}$ space $[1,7]$. It is convenient for augmenting existing geographical maps with tactile information layers or guiding the user to the location of an interface element as suggested in [1]. So far, most previous research on tactile perception has only considered ultrasonic physical manifestation as an output modality $[1,19]$. However, Obrist et al.'s interviews on users' blinded tactile experiences with ultrasonic haptics suggest that participants felt sensations similar to interacting with physical objects such as "pieces of foam", "soft brushes" or "pins and needles" when their hand was stimulated by ultrasonic focal points [13]. 
We believe that providing tactile sensations in mid-air, produced by such ultrasonic haptics systems, have the potential to substitute physical widgets such as buttons, sliders or dials by virtual widgets floating in the air. It would not only offer a haptic input system with complementary discrete or continuous physical commands on top of existing physical inputs but also a reconfigurable command space at will to fulfil users' expectations depending on the current task they are dealing with. While designing such widgets raises several interesting challenges on their operating and users' perception, we must start by investigating the spatial localization of ultrasonic haptic virtual widgets. More precisely, we are interested in understanding whether it is possible to spatially reach these widgets without visual feedback, as visual attention may not be always available for secondary tasks.

The rest of this paper is organized as follows. We first review the relevant work on virtual widgets, mid-air freehand gesture haptic feedback, and introduce ultrasonic virtual widgets. We then report a user study that investigates the spatial localisation of virtual widgets with and without ultrasonic haptic cues. Our results show that users perform slightly faster and were more accurate when locating ultrasonic haptic targets in mid-air. We finally compare our results with previous work that has investigated the spatial resolution of passive localisation of ultrasonic haptic feedback.

\section{RELATED WORK}

\section{A. Locating virtual widgets}

Virtual reality research offers many interaction techniques to select objects in a 3D space, such as ray casting or volume selection techniques [16]. However, these techniques remain imprecise for selecting very small objects, since the selection is happening from a distance. To cope with the lack of haptic feedback, researchers have investigated the role of visual cues for direct touch of intangible display. For example, Chan et al. used a representation of a shadow of the hand interacting with the system to improve the performance of a target acquisition task [3]. Their results show that visual and audio cues improved acquisition time, even if the visual feedback was hidden by the dominant hand or occluded visual content when plainly visible. Interestingly, participants that performed well after minimal practice did not need the visual and audio feedback anymore.

Spatial awareness alone can support the spatial localisation of virtual targets $[6,10]$. Virtual shelves are a system that triggers command shortcuts by orienting a phone towards a specific direction within the circular hemisphere in front of the user [10]. The system was able to offer 28 selectable regions of the middle hemisphere in front of its users. However, users that were blindfolded during an experiment were less precise when targets were situated towards the left or right of the hemisphere. Also, the targets were large, limiting the number of available inputs and precise accuracy was not required, as targets covered an area of space.
Imaginary interfaces have shown that short-term working memory enables spatial interaction without visual feedback [6]. In a pointing task experiment, users were able to acquire virtual points by their coordinates in a $2 \mathrm{D}$ vertical plane in front of the user. However, the interaction required users to continuously manifest a visual referential defined by a specific ' $L$ ' gesture, executed by their non-dominant hand. In addition, Gustafson et al.'s results suggest that pointing precision decreases as the interaction moves away from the origin of the referential. While spatial awareness provides advantages for spatial localisation without visual feedback, the precision of the interaction remains limited.

\section{B. Haptic feedback for mid-air gestures}

Providing haptic feedback for mid-air gestures has gained attention from HCI researchers $[7,8,15]$. Using piezoelectric actuators, Kim et al. provided participants with haptic feedbacks to inform them of the start, the end and the recognition states of a gesture [8]. Comparing with a situation where no feedback was provided, they showed that haptic feedback increased the accuracy and reduced the trajectory length of the gesture, and potentially users' fatigue. However, participants required to be instrumented with wired actuators, which could be cumbersome for everyday tasks.

Another technique to provide tactile feedback for 3D gestures is blowing air vertices on the skin [15]. These systems, which do not require any instrumentation of the users, are capable of deliver haptic feedback to long distances up to 2.5 meters [5]. However, when aiming at different parts of the body with air vortices, Gupta et al. could not reach a resolution better than $10 \mathrm{~cm} \mathrm{[5].}$

Ultrasounds have been investigating as a means to convey haptic feedback for mid-air gestures [2,7]. For example, the UltraHaptics techonology takes advantage of acoustic radiation pressure [2]. By focusing a "beam" of sound waves on a particular location at the surface of the skin, a tactile sensation is created when the amplitude-modulated beam is reflected off the skin, producing a perceivable haptic sensation. By tracking users' hands with a suitable sensor, ultrasound haptics can be projected directly onto users' hands as they perform mid-air gestures. This technology is able to produce ultrasonic focal points with a $3 \mathrm{D}$ spatial precision of one millimetre [2], which is more precise than air vortices based technologies such as AIREAL [15]. In addition, like AIREAL ultrasonic based haptic systems do not require users to be instrumented [4].

Previous research has shown that haptic feedback has the potential to improve mid-air gestural interaction [8]. In this paper, we focus our research on the localisation of virtual widgets that are reachable by hands. Therefore, ultrasonic haptic feedback technology seems to be the most appropriate. In the next section, we review the literature that has studied the perception of ultrasonic haptic feedback. 


\section{Ultrasonic haptic feedback}

Users can perceive static ultrasonic points and dynamic motion across their hand [2,19]. Carter et al. have shown that user were able to perceive multiple ultrasonic focal points simultaneously, depending on the frequency they were produced at and their distance of separation [2]. Wilson et al. investigated the passive localization of ultrasonic points and the perception of movement across the hand [19]. In their study, they had participants position their hand on a table with their palm pointing upwards. Above the hand, an ultrasonic array produced static points, at different locations across the fingers and palm, and a sequence of two to five points in straight lines eliciting apparent motion. They found that larger distances, a higher number of points, and longer point duration improve the perception, the localization and the quality of perceived movement. Their results also suggest that the minimum spatial resolution to display a point across the hand is $1.5 \times 2 \mathrm{~cm}$. Therefore, they recommend designing virtual objects with a minimum size of $2 \mathrm{~cm}^{2}$.

It is also worth noticing Hoshi et al.'s study on active localisation of ultrasonic haptic points [7]. In their experiment, they asked participants to follow a point displacement with the palm of their hand from the centre of their array towards one of the 8 cardinal directions. They report a mean error distance of $8.9 \mathrm{~mm}(\mathrm{SD}=7.4 \mathrm{~mm}$ ) suggesting that users can stabilize their hand within $16.3 \mathrm{~mm}$ from a fixed focal point.

Studies on perception and localisation of ultrasonic haptic feedback have mainly considered passive exploration i.e. haptic manifestation produced on motionless body parts. More research is needed to understand active exploration of ultrasonic physical sensations in mid-air i.e. when haptic perception is combined with motion of the hand.

\section{ULTRASONIC INPUT}

Ultrasound haptics has mostly been used as feedback for mid-air gestures. However, it has also the potential for creating haptic widgets in mid-air, which users can interact without visual attention. Instead, users can use active haptic exploration and their spatial awareness for locating and interacting with these virtual haptic widgets. We call them ultrasonic haptic widgets.

Ultrasonic haptic widgets build on the concept of Imaginary Interfaces [6] by providing invisible but physically perceptible widgets in mid-air. For example, combining multiple ultrasonic focal points into complex structures would allow designers to create different sets of widgets which can execute commands such as virtual buttons (Figure 1), or control continuous parameters such as virtual sliders or dials. Since these widgets are virtual, they can be moved and reconfigured at will to fit any type of task, as needed. Because ultrasonic haptic widgets are tactually perceptible, no specific gestures or visual cues should be required to locate and interact with them. While manipulating virtual widgets formed of several haptic ultrasonic points presents an interesting challenge, the localisation of these virtual forms of physical widgets is just as essential. However the performance of such task still remains unknown. As a starting point, we begin to investigate the spatial localization of such widgets in space, including in the absence of visual feedback. While previous work has studied the spatial resolution of ultrasonic feedback across motionless hands, we aim to determine the spatial resolution of ultrasonic widgets during active tactile exploration, which has not been previously studied yet.

\section{USER STUdy: LOCALIZING VIRTUAL WIDGETS}

The purpose of this study was to investigate how well users can locate mid-air ultrasonic haptic widgets. Our hypotheses were:

- H1: locating visual points will be faster than acquiring haptic points;

- H2: locating ultrasonic points will be more accurate than visual points;

- H3: locating combined visual and haptic points will be faster than acquiring haptic points and more accurate than locating visual points;

- H4: the spatial resolution of active touch of ultrasonic points will be finer than for passive touch.

\section{A. Task}

Our tasks required users to locate a point in a 2D space, while standing in front of a desk. A display, keyboard and ultrasound haptics device were positioned on the desk (Figure 3). Although ultrasound focal points can be positioned in 3D space, we fixed the height of the target point to $20 \mathrm{~cm}$ above the surface of our device. We chose a fixed height to focus on interaction on a $2 \mathrm{D}$ plane simulating a single layer of virtual widgets, as previous research has reported large errors for $3 \mathrm{D}$ acquisition for height inferior to $10 \mathrm{~cm} \mathrm{[1].} \mathrm{We}$ plan to investigate the case of multi-layers in future research. A fixed height of $20 \mathrm{~cm}$ also allowed participants to acquire targets in their comfort zone [9] without creating any discomfort or fatigue. A single point represents the smallest virtual widget generated by our device, which is $8.4 \mathrm{~mm}$ diameter as we are using $40 \mathrm{kHz}$ ultrasound. We placed a marker on the distal inter-phalangeal joint of the middle finger. This location was selected based on informal feedbacks we received during pilot studies. The location on the surface of the finger underneath the marker seemed to be very sensitive to ultrasonic feedback for several users. The marker was used as a point of reference for participants to acquire the target. We asked participants to match the 2D position of the marker to the 2D position of the target point as fast and accurately as possible. Each trial started when the participant hit the space bar of the keyboard in front of them. Then, participants had to locate the target above the array with their finger in each condition. When the marker was aligned with the target's $\mathrm{x}$ and $\mathrm{y}$ coordinates, participants pressed the space bar to confirm their selection and the trial ended.

\section{B. Experimental design}

The study had one independent variable with three levels: visual, haptic and visual and haptic. Before each trial, the target coordinates were randomly computed in a square of 
$130 \mathrm{~mm} * 130 \mathrm{~mm}$ with a precision of $1 \mathrm{~mm}$. The origin of this space was located above the centre of the ultrasound haptics array. We did not use the entire available space above the array, as ultrasonic focal points are stronger when produced in the centre area [19]. In the visual condition, participants were shown an empty square representing the horizontal surface area of the array, the space within which the target would be positioned (Figure 2). This representation was selected to give enough information for users to raise their spatial awareness as suggested in [6]. When the trial started, a red cross was positioned inside the square at the target location. No feedback was provided for the participants' finger motion. In the haptic condition, the target location was indicated by an ultrasonic focal point produced by our array at an amplitude-modulated at a frequency of $250 \mathrm{~Hz}$ (Figure 2). Only the $\mathrm{x}$ and $\mathrm{y}$ coordinates of the target varied. All target points were generated on a plane $200 \mathrm{~mm}$ above the array. A visual cue on the screen bezel indicated the height of the plane where the ultrasonic haptic points were produced. To mask any auditory cues produced by the ultrasonic array that could have assisted the localization of the targets, participants wore a pair of in-ear headphones and listened to pink noise.

The study was completed when the participant had acquired 300 targets. Targets were divided into 10 blocks of 10 trials for each condition, giving participants time to rest between groups of trials. Participants were asked to practice 10 trials before each condition, to familiarize themselves with the system and the stimuli. In total, we collected 5400 trials (18 participants $\mathrm{x} 3$ conditions $\mathrm{x} 100$ trials).

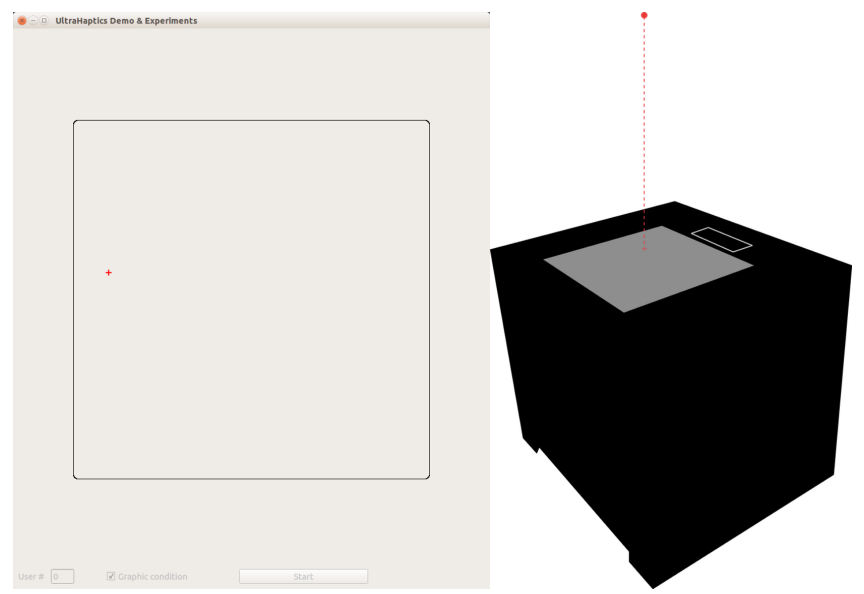

Figure 2. Stimuli presented during the experiment. The figure on the left is the visual stimulus. The figure on the right is the haptic stimulus.

The experimental conditions were fully counter-balanced to reduce bias and ordering effects as much as possible. For each trial, we measured the precision of the selection and the time of selection. The precision error was defined by the Euclidian distance between the marker position on the middle finger at the time of the trial validation and the target position. We also measured the distance from the target location along the $\mathrm{x}$ - and the $\mathrm{y}$-axis of the referential. The time of selection was the elapsed time between two consecu- tive space bar press events marking the start and the end of the trial.

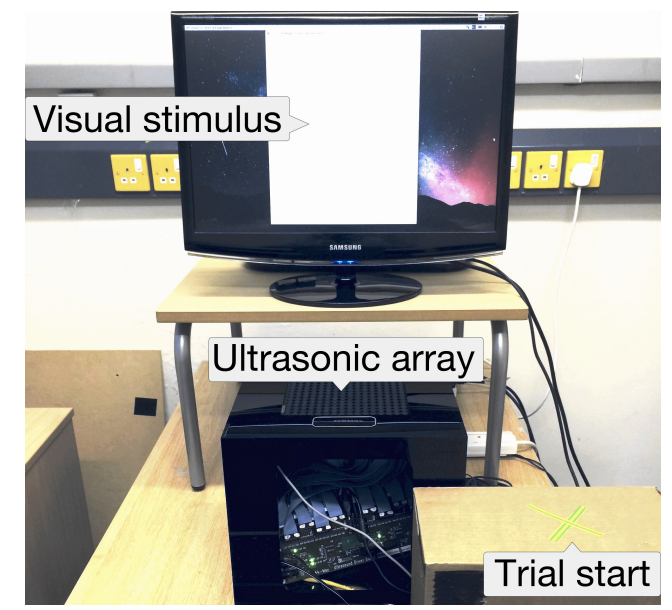

Figure 3. Experimental setup. Each trial starts with the hand on the cross mark. The localisation of the target point is made above the ultrasonic array.

\section{Apparatus}

The experiment software was running on a desktop PC equipped with an Intel Core i7-920 processor and an Nvidia GTX770 graphics card. A Leap Motion ${ }^{\star}$ sensor was used to capture the participants' hand and finger movements. This sensor allows fingers tracking with an average accuracy of $0.7 \mathrm{~mm}$ [18]. Ultrasonic focal points were generated by an Ultrahaptics device [2], which was equipped with an array of $16 \times 16$ muRata MA40S4S transducers of $10 \mathrm{~mm}$ diameter each. Graphical stimuli were displayed using the $\mathrm{C}++\mathrm{Qt}$ library (Figure 3).

\section{Participants}

Eighteen right-handed participants ( 5 females) aged between 18 to 31 years $($ Mean $=21.83, \mathrm{SD}=3.55)$ were recruited at our institution and were given $£ 6$ for their participation in the study, which took approximately 60 minutes to complete.

\section{E. Results}

We removed $2.4 \%$ of outliers from the data. A trial was considered as an outlier when the time or the precision error was more than three standard deviations (SD) from the overall mean time or the overall precision error. A log transformation was applied to the data before conducting any ANOVAs.

The mean time for acquiring the target reached $2.281 \mathrm{~s}$ $(\mathrm{SD}=0.704 \mathrm{~s})$ for the visual condition, 2.797s $(\mathrm{SD}=0.836 \mathrm{~s})$ for the haptic condition and $2.220 \mathrm{~s}(\mathrm{SD}=0.829 \mathrm{~s})$ when participants were presented with both visual and haptic cues (Figure 4). A one-way repeated-measures within-subjects ANOVA showed a significant effect $\left(\mathrm{F}_{2,17}=5.602, \mathrm{p}<0.01\right)$. Post hoc pairwise comparisons with Bonferroni correction revealed that participant were significantly faster in the visual $(\mathrm{p}<0.05)$ and in the visual and haptic $(\mathrm{p}<0.05)$ than in the haptic condition. No significant difference was found between the visual and the visual and haptic condition.

\footnotetext{
* https://www.leapmotion.com
} 


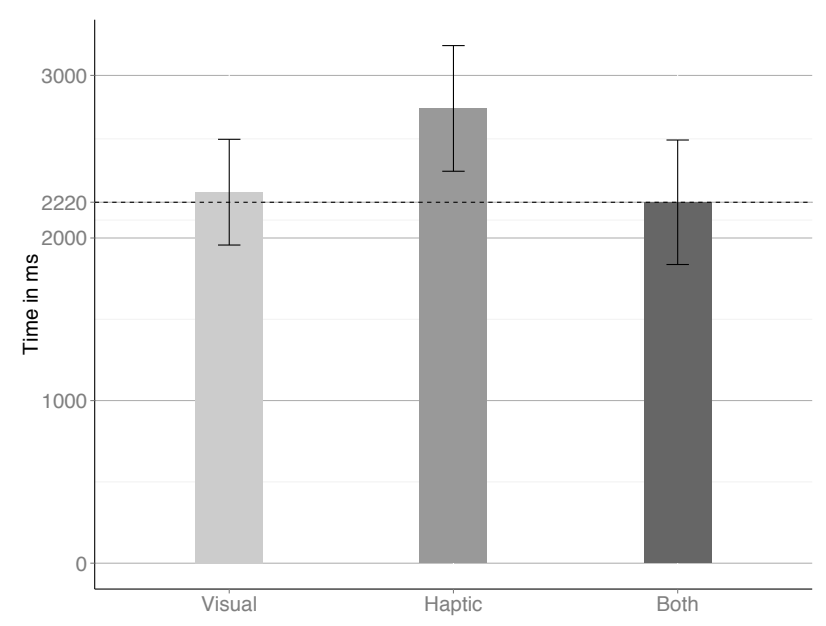

Figure 4. Mean execution time (ms). Error bars represent $95 \%$ confidence intervals. The dot line represents the fastest average time.

The mean precision error was $20.7 \mathrm{~mm}(\mathrm{SD}=6 \mathrm{~mm})$ for the visual condition, $14.1 \mathrm{~mm}(\mathrm{SD}=4.3 \mathrm{~mm})$ for the haptic condition and $11 \mathrm{~mm}(\mathrm{SD}=2.7 \mathrm{~mm})$ when presented with both haptic and visual cues (Figure 5). There was a significant effect of the type of stimulus on the mean Euclidian distance error $\left(\mathrm{F}_{2,17}=26.375, \mathrm{p}<0.001\right)$. Post-hoc pairwise t-tests with Bonferroni correction exhibited a significant difference between visual and haptic $(\mathrm{p}<0.005)$, between visual and visual and haptic $(\mathrm{p}<0.001)$, and haptic and visual and haptic conditions $(\mathrm{p}<0.005)$.

The mean precision error along the $\mathrm{x}$-axis was $9.2 \mathrm{~mm}$ for the visual condition, $7.5 \mathrm{~mm}$ for the haptic condition and 5.9 $\mathrm{mm}$ when presented with both haptic and visual cues. Along the y-axis, it was $16.6 \mathrm{~mm}$ for the visual condition, $10.4 \mathrm{~mm}$ for the haptic condition and $8.1 \mathrm{~mm}$ when presented with both haptic and visual cues. There was a significant difference on the error distance along the $\mathrm{x}$ axis $\left(\mathrm{F}_{2,17}=11.56\right.$, $\mathrm{p}<0.001)$ and along the $\mathrm{y}$ axis $\left(\mathrm{F}_{2,17}=18.074, \mathrm{p}<0.001\right)$. A post-hoc pairwise t-test with Bonferroni correction revealed a significant difference between visual and visual and haptic $(\mathrm{p}<0.001)$ and between haptic and visual and haptic $(\mathrm{p}<0.05)$ conditions along the $\mathrm{x}$-axis. There was also a significant difference between visual and haptic $(\mathrm{p}<0.005)$, visual and visual and haptic $(\mathrm{p}<0.001)$ and between haptic and visual and haptic $(\mathrm{p}<0.05)$ conditions along the $\mathrm{y}$-axis.

\section{F. Discussion}

Hypotheses $\mathrm{H} 1$ and $\mathrm{H} 2$ were supported by the results. Although there was not a significant difference between the visual and the visual and haptic condition in execution time, we found that participants were faster in the visual condition than in the haptic condition. When presented with haptic feedback alone, we observed that participants were exploring the space above the array to locate the ultrasonic focal point with their hand. In addition, participants adjusted the middle finger position to match the target point position before validating the selection. These actions consequently delayed the selection. In the case of the visual condition, most of the participants directed their hand straight away to the target. Since no haptic feedback was provided, they did not feel the need to adjust the position of their finger and were less precise when locating visual than locating haptic points.

Hypothesis H3 was also supported. In the haptic and visual condition, participants were significantly faster than in the haptic condition and significantly more accurate than in the visual condition. While the visual alone condition represents the case of the user being spatially aware of spatial location of virtual widgets in space, the haptic and visual condition represents a situation where a user would already know the rough location of the widgets and would use touch to find its exact position.

Hypothesis $\mathrm{H} 4$ was not (fully) verified. The mean distance error varied from $5.9 \mathrm{~mm}$ to $9.2 \mathrm{~mm}$ along the $\mathrm{x}$-axis and from $8.1 \mathrm{~mm}$ to $16.6 \mathrm{~mm}$ along the $\mathrm{y}$-axis (Table 1 ). While the largest error was found for the condition with visual cues alone, the smallest error for both axes was found when visual and haptic cues were presented to participants. Surprisingly, the results suggest that active localisation (i.e. exploration by moving the hand) is worse than passive localisation (i.e. hand still) [19]. For haptic alone, the average error was $5.6 \mathrm{~mm}$ higher than the measure reported for passive localisation $(8.5 \mathrm{~mm}, \mathrm{SD}=6.8 \mathrm{~mm})$ [19]. Interestingly, when participants knew the location of the target, the average error decreases to $11 \mathrm{~mm}$ showing an improvement of more than $20 \%$ from our results in the haptic alone condition and almost $50 \%$ in the visual alone condition.

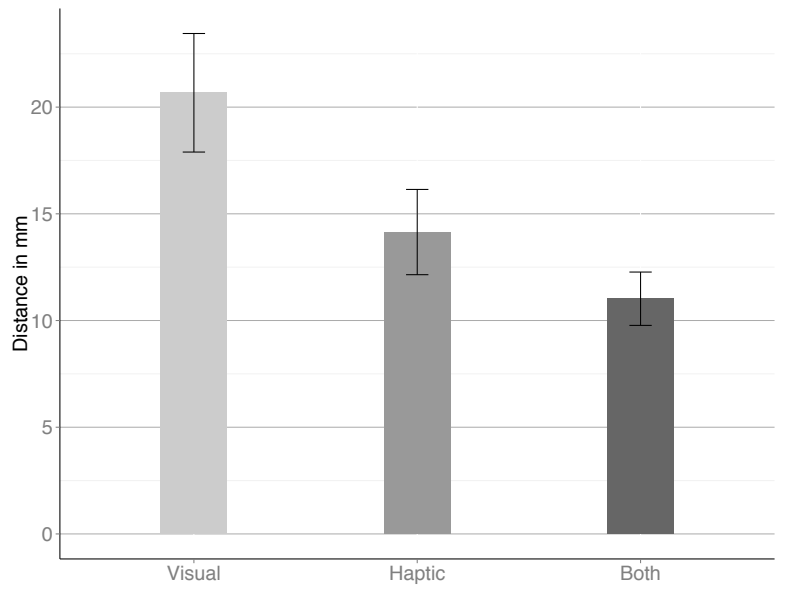

Figure 5. Mean Euclidian distance error (mm). Error bars represent 95\% confidence intervals

When dissociating measures along the $\mathrm{x}$ - and the $\mathrm{y}$-axis, the results match the results reported in [19]. In general, the mean error along the $\mathrm{y}$-axis was higher than along the $\mathrm{x}$-axis.

For example, in the haptic condition, we found that the error along the $y$-axis was a little less than $3 \mathrm{~mm}$ worse than along the $\mathrm{x}$-axis. This result suggests that active localisation of static ultrasonic haptic point has the same level of reliability than passive localisation. Interestingly, this measure decreases to $2.2 \mathrm{~mm}$ with visual and haptic cues, suggesting an improvement of more than $25 \%$ when the user is aware of the location of the target. 
TABLE I. MEAN ERROR DISTANCES BY EXPERIMENTAL CONDITION

\begin{tabular}{|c|c|c|c|}
\hline & \multicolumn{3}{|c|}{ Mean distance errors in $\mathbf{m m}$} \\
\hline & Condition & Mean & $\begin{array}{l}\text { Standard } \\
\text { deviation }\end{array}$ \\
\hline \multirow{3}{*}{$\begin{array}{c}\text { Euclidian } \\
\text { distance }\end{array}$} & Visual & 20.7 & 6 \\
\hline & Haptic & 14.1 & 4.3 \\
\hline & Visual \& Haptic & 11 & 2.7 \\
\hline \multirow{3}{*}{$\begin{array}{l}\text { Distance along } \\
\text { the } x \text {-axis }\end{array}$} & Visual & 9.2 & 2.4 \\
\hline & Haptic & 7.5 & 2.2 \\
\hline & Visual \& Haptic & 5.9 & 2.0 \\
\hline \multirow{3}{*}{$\begin{array}{c}\text { Distance along } \\
\text { the y-axis }\end{array}$} & Visual & 16.6 & 6.8 \\
\hline & Haptic & 10.4 & 3.9 \\
\hline & Visual \& Haptic & 8.1 & 2.2 \\
\hline
\end{tabular}

Error location differed depending on the conditions (Table 1). Interestingly, the standard deviation was highest in the visual condition and lowest in the visual and haptic condition along the $\mathrm{x}$ - and the $\mathrm{y}$-axis, suggesting that the results were more consistent when participants were presented with haptic feedback along with a visual reference frame. The findings presented in this paper indicate that localisation is theoretically possible at a spatial resolution of $11.6 \mathrm{~mm} \mathrm{x}$ $23.5 \mathrm{~mm}$ when only a visual reference is provided, $9.8 \mathrm{~mm} \mathrm{x}$ $14.3 \mathrm{~mm}$ when users solely rely on haptic exploration, and $7.9 \mathrm{~mm} \times 10.3 \mathrm{~mm}$ when ultrasonic haptic feedback is combined with spatial awareness. Depending on the condition, these distances would correspond to a spatial resolution of $14 \times 6$ (visual), $17 \times 11$ (haptic) or $20 \times 15$ points (visual and haptic) on our current ultrasonic haptic display $(160 \mathrm{~mm} x$ $160 \mathrm{~mm}$ ). Finally, in the situation of active exploration, the virtual object size should not be smaller than $5.5 \mathrm{~cm}^{2}$ if only visual feedback is provided, $2 \mathrm{~cm}^{2}$ if users need to explore the space with his hand; and $1 \mathrm{~cm}^{2}$ if user has access to both a visual reference and ultrasonic haptic feedback.

\section{CONCLUSION}

This paper has investigated the localisation of ultrasound haptic target points in mid-air. In future work, we plan to broaden our study to investigate spatial localisation of virtual widgets on multi-layered interfaces (i.e. in three dimensions). In addition, we plan to design ultrasonic haptic widgets and investigate how they can be discriminated and manipulated through gesture. We will also evaluate their effectiveness for human-computer interaction.

When locating virtual widgets in $2 \mathrm{D}$, our results show that the precision significantly improves when providing ultrasonic cues in addition to spatial awareness. When users have a visual reference for the location of a virtual widget, ultrasonic haptics can improve the precision of reaching a widget by $50 \%$. In addition, to be correctly perceived, the minimum distance along axes between two different virtual points can descend to $7.9 \mathrm{~mm} \times 10.3 \mathrm{~mm}$, allowing users to interact with virtual widgets with a minimum size of $1 \mathrm{~cm}^{2}$.

\section{ACKNOWLEDGMENT}

This research was funded by EPSRC grant EP/J005312/1. The authors would also like to thank Graham Wilson, Euan Freeman and Alexander Ng.

\section{REFERENCES}

[1] Bruder G., Steinicke F. and Stuerzlinger W. "Touching the Void Revisited: Analyses of Touch Behavior on and above Tabletop Surfaces". In Proc. of INTERACT 2013, Lecture Notes in Computer Science Volume 8117, 2013, pp. 278-296.

[2] Carter T., Seah S. A., Long B., Drinkwater B. and Subramanian S. "UltraHaptics: multi-point mid-air haptic feedback for touch surfaces". In Proc. of UIST '13. ACM, New York, NY, USA, 2013, pp. 505-514.

[3] Chan L.-W., Kao H.-S., Chen M. Y., Lee M.-S., Hsu J. and Hung Y.P. "Touching the void: direct-touch interaction for intangible displays". In Proc. CHI '10. ACM, New York, NY, USA, 2010, pp. 2625-2634.

[4] Freeman E., Brewster S., and Lantz V. "Tactile Feedback for AboveDevice Gesture Interfaces: Adding Touch to Touchless Interactions". In Proc. of ICMI '14. ACM, New York, NY, USA, 2014, pp. 419426.

[5] Gupta S., Morris D., Patel S. N. and Tan D. "AirWave: non-contact haptic feedback using air vortex rings”. In Proc. of UbiComp '13. ACM, New York, NY, USA, 2013, pp. 419-428.

[6] Gustafson S., Bierwirth D. and Baudisch P. "Imaginary interfaces: spatial interaction with empty hands and without visual feedback". In Proc. Of UIST '10. ACM, New York, NY, USA, 2010, pp. 3-12.

[7] Hoshi, T.; Takahashi, M.; Iwamoto, T.; Shinoda, H., "Noncontact Tactile Display Based on Radiation Pressure of Airborne Ultrasound, IEEE Transactions on Haptics, vol.3, no.3, 2010, pp.155-165.

[8] Kim K., Kim J., Choi J., Kim J., Lee S., Depth Camera-Based 3D Hand Gesture Controls with Immersive Tactile Feedback for Natural Mid-Air Gesture Interactions. Sensors 2015, 15, pp. 1022-1046.

[9] Kölsch M., Beall A. C. and Turk M. "The Postural Comfort Zone for Reaching Gestures". In Proc. of the Human Factors and Ergonomics Society Annual Meeting, 2003, vol. 47, pp. 787-791,

[10] Li F. C. Y., Dearman D., and Truong K. N. "Virtual shelves: interactions with orientation aware devices". In Proc. of UIST '09. ACM, New York, NY, USA, 125-128.

[11] Long B., Seah S. A., Carter T. and Subramanian S. "Rendering volumetric haptic shapes in mid-air using ultrasound". ACM Trans. Graph. 33, 6, Article 181. ACM, New York, NY, USA, 2014.

[12] Marquardt N., Jota R., Greenberg S. and Jorge J. "The Continuous Interaction Space: Interaction Techniques Unifying Touch and Gesture On and Above a Digital Surface". In Proc. of INTERACT 2011. (Lisbon, Portugal), September 2011, pp. 5-9.

[13] Obrist M., Seah S. A. and Subramanian S. "Talking about tactile experiences". In Proc. of CHI '13. ACM, New York, NY, USA, 2013, pp. 1659-1668.

[14] Rümelin, S., Marouane, C. and Butz, A. "Free-hand pointing for identification and interaction with distant objects". In Proc. of AutomotiveUI '13. ACM, New York, NY, USA, 2013, pp. 40-47.

[15] Sodhi R., Poupyrev I., Glisson M. and Israr A. "AIREAL: interactive tactile experiences in free air". ACM Trans. Graph. 32, 4, Article 134, 2013.

[16] Steed, A., "Towards a General Model for Selection in Virtual Environments," IEEE Symposium on 3D User Interfaces, 3DUI 2006, pp. 25-29

[17] Taylor S., Keskin C., Hilliges O., Izadi S. and Helmes J. “Type-hoverswipe in 96 bytes: a motion sensing mechanical keyboard". In Proc. of CHI '14. ACM, New York, NY, USA, 2014, pp. 1695-1704.

[18] Weichert, F.; Bachmann, D.; Rudak, B.; Fisseler, D. Analysis of the Accuracy and Robustness of the Leap Motion Controller. Sensors 2013, 13, 6380-6393.

[19] Wilson G., Carter T., Subramanian S. and Brewster S. A. Perception of ultrasonic haptic feedback on the hand: localisation and apparent motion. In Proc. of CHI '14. ACM, New York, NY, USA, 2014, pp. 1133-1142. 